\title{
No association between latent toxoplasmosis and multiple body measures in U.S. adults
}

\author{
${ }^{1}$ Department of Psychology, Brigham Young University, Provo, Utah, USA; \\ ${ }^{2}$ The Neuroscience Center, Brigham Young University, Provo, Utah, USA; \\ ${ }^{3}$ Department of Sociology, Brigham Young University, Provo, Utah, USA
}

Andrew N. Berrett ${ }^{1}$, Shawn D. Gale ${ }^{1,2}$, Lance D. Erickson ${ }^{3}$, Bruce L. Brown ${ }^{1}$ and Dawson W. Hedges ${ }^{1,2}$

\begin{abstract}
Toxoplasma gondii (Nicolle et Manceaux, 1908) is an intracellular parasite that can cause ongoing latent infection persisting for the duration of a non-definitive host's life. Affecting approximately one-third of the world's population, latent toxoplasmosis has been associated with neuropsychological outcomes and a previous report suggested an association between latent toxoplasmosis and adult height. Given the large number of people with latent toxoplasmosis and its potential associations with human height, we sought to better understand the association between latent toxoplasmosis and human morphology by evaluating seropositivity for $T$. gondii and multiple body measures reported in the National Health and Nutrition Examination Survey III (NHANES III) and in the more recent continuous NHANES data sets from the United States Centers for Disease Control and Prevention for which data on T. gondii are available. In these analyses, latent toxoplasmosis was not associated with any of the body measures assessed in the NHANES datasets even after taking into account interactions between latent toxoplasmosis and testosterone suggesting that in these samples, latent toxoplasmosis is not associated with adult morphology including height.
\end{abstract}

Keywords: Toxoplasma gondii, height, testosterone, BMI

This article contains supporting information (Tables S1-S3) online at http://folia.paru.cas.cz/suppl/2016-63-034.pdf

Latent infection with the intracellular protozoan parasite Toxoplasma gondii (Nicolle et Manceaux, 1908) is prevalent in an estimated $30 \%$ of the world's population with considerable variation between countries (Jones et al. 2014). Moreover, latent toxoplasmosis can persist for the life of the host. Although felids are the primary host for T. gondii, humans may become infected via exposure to oocytes by consuming contaminated water or foods such as improperly cooked meats (Finsterer and Auer 2013).

Associations between latent toxoplasmosis and impairments in cognitive function, personality changes and psychiatric disease (Flegr et al. 2000, Mortensen et al. 2007, Gale et al. 2015) suggest that latent toxoplasmosis could affect brain structure and function. Further, latent toxoplasmosis has been associated with human morphology. Нypothesising that men with high testosterone concentrations would be more likely to engage in behaviour increasing their chances of exposure to T. gondii, Flegr et al. (2005) found significant differences between infected and uninfected men in standing height and the ratio between the lengths of the second and fourth digits of the hand, the latter thought to be due to prenatal androgenisation affecting subsequent morphology and behaviour related to risk factors for exposure to T. gondii (see Flegr et al. 2008).
Additional evidence for an association between toxoplasmosis and human morphology is found in a study demonstrating that women rate the faces of men with latent toxoplasmosis as more dominant and masculine than they do the faces of men without latent toxoplasmosis (Hodková et al. 2007). A possible explanation of putative associations between latent toxoplasmosis and human morphology is the increased testosterone found in men with latent toxoplasmosis in some but not all studies (Flegr 2013). Finally, infection with $T$. gondii may affect metabolism. For example, a study by Kaňková et al. (2015) found that pregnant mothers with latent toxoplasmosis had higher blood glucose levels and were thereby at greater risk of developing gestational diabetes.

Given the high prevalence of latent toxoplasmosis and its reported associations with human cognitive function and morphology whether via testosterone influencing behaviour related to exposure to $T$. gondii or direct effects of latent toxoplasmosis itself on human morphology, we sought to further investigate the relationship between latent toxoplasmosis and human morphology using multiple body measures in addition to height in both women and men in large, chronologically distinct adult samples. 
Utilising the publically available National Health and Nutrition Examination Surveys III (NHANES III) and the continuous NHANES data sets made available by the Centers for Disease Control and Prevention (CDC), we evaluated associations between latent toxoplasmosis and multiple body measures while controlling for variables such as sex, socioeconomic status, educational attainment and race-ethnicity that could confound apparent associations between latent toxoplasmosis and human morphology. We also tested for interaction effects between latent toxoplasmosis and testosterone concentration in the prediction of each body measure included in this study.

\section{MATERIALS AND METHODS}

\section{Study sample}

The NHANES is an ongoing, nation-wide survey conducted by the National Center of Health Statistics at the CDC in the United States to obtain demographic, medical and other data from randomly chosen samples across the nation. The NHANES III was conducted from 1988 to 1994 and the continuous NHANES has been actively collecting data since 1999 . In the present study, we obtained data from the NHANES III and the 1999-2004 and 20092012 data cycles of the continuous NHANES. The NHANES data are designed to be representative of the U.S. population through the use of complex, multi-stage sampling and statistical weighting. As our goal was to investigate the potential effects of infection with $T$. gondii on post-developmental, adult body measures, we chose to only include subjects between the ages of 21 to 49 years in all three NHANES data sets. By doing so, we could reasonably expect that all included subjects had likely achieved full development but also had not yet experienced significant age-related changes in body size due to physical deterioration disorders such as osteoporosis. We did not include in the analyses subjects who were missing data for any of the variables necessary for this study. In total, we included 7118 subjects from the NHANES III, 4008 from the 1999-2004 continuous NHANES and 4036 from the 2009-2012 continuous NHANES data sets.

\section{Serology of Toxoplasma gondii}

In the NHANES samples, CDC lab technicians determined the presence of latent toxoplasmosis using an indirect enzyme immunoassay in which the optical density of a serum sample was compared to a World Health Organization calibrated standard curve. As the clinical criterion for seropositivity has been regularly updated over the past two decades, the minimum immunoglobulin $\mathrm{G}(\mathrm{IgG})$ titre values required for diagnosis of latent toxoplasmosis was different across the NHANES data sets due to differences between manufacturer guidelines of the different assays. In the NHANES III and 1999-2004 continuous NHANES data sets, samples with test results below 7 international units (IU/ml) were considered negative for infection, while results 7 $\mathrm{IU} / \mathrm{ml}$ and above were considered positive (Gunter et al. 1996). For results in the 2009-2012 continuous NHANES data set, values below $32 \mathrm{IU} / \mathrm{ml}$ were considered to be negative for $T$. gondii and positive if at or above $33 \mathrm{IU} / \mathrm{ml}$. In our models, we coded subjects with a positive titre result as 1 and those with a negative result as 0 . As the test only sampled IgG antibody concentration, we could not determine the time the initial infection occurred.

\section{Body measures}

We used all available body measures in both the NHANES III and continuous NHANES data sets in our analyses. However, there were some differences in which body measures were available in each data set. From NHANES III, we used standing height $(\mathrm{cm})$, body weight $(\mathrm{kg})$, body mass index (BMI, calculated by weight in $\mathrm{kg} /$ height in $\mathrm{m}^{2}$ ), waist circumference $(\mathrm{cm})$, upper-leg length $(\mathrm{cm})$, arm circumference $(\mathrm{cm})$, upper-arm length $(\mathrm{cm})$, thigh circumference $(\mathrm{cm})$, waist-to-hip ratio (waist circumference/hip circumference), biacromial breadth $(\mathrm{cm})$, biiliac breadth $(\mathrm{cm})$, elbow breadth $(\mathrm{cm})$, wrist breadth $(\mathrm{cm})$ and buttocks circumference $(\mathrm{cm})$.

From the 1999-2004 continuous NHANES data sets, we used standing height $(\mathrm{cm})$, body weight $(\mathrm{kg})$, BMI (see formula above), waist circumference ( $\mathrm{cm})$, upper-leg length $(\mathrm{cm})$, arm circumference $(\mathrm{cm})$, upper-arm length $(\mathrm{cm})$, thigh circumference (cm) and calf circumference. Finally, from the 2009-2012 continuous NHANES data sets, we used standing height $(\mathrm{cm})$, body weight $(\mathrm{kg})$, BMI (see formula above), waist circumference $(\mathrm{cm})$, upper-leg length $(\mathrm{cm})$, arm circumference $(\mathrm{cm})$ and upper-arm length $(\mathrm{cm})$.

\section{Testosterone}

Sufficient testosterone data to include as a variable in statistical models was only available in the 2011-2012 continuous NHANES data set. Other NHANES cycles such as the 1999-2004 data sets included testosterone data but only for a very small subsample of subjects and thus significantly increased risk of statistical error. In the 2011-2012 NHANES data set, CDC technicians determined total serum testosterone $(\mathrm{ng} / \mathrm{dl})$ via isotope dilution liquid chromatography tandem mass spectrometry. This method is based on the National Institute for Standards and Technology's reference method and is optimised for higher sample throughput.

\section{Covariates}

We included a number of covariates in our analyses to control for potential confounding. Categorical covariates were race-ethnicity (non-Hispanic white, non-Hispanic black, Mexican American, other Hispanic, other), and sex. Continuous covariates included age and poverty-to-income ratio (PIR, defined as a ratio between the primary household income and the federally established poverty level at the time of data collection). Educational attainment was measured as completed years of schooling in the NHANES III data set but was instead reported in a categorical format (less than high school, high school and more than high school) in the 1999-2004 and 2009-2012 continuous NHANES data sets. We also included seropositivity for cytomegalovirus (CMV) as a categorical control of potential effects due simply to the presence of latent infections according to the method used by Flegr et al. (2005). However, data for CMV were not available in the 2009-2012 NHANES data set and were therefore excluded as a covariate in analyses using those data.

\section{Statistical analysis}

We used Stata release 14.1 for all analyses and used the svy prefix to account for the complex, multi-stage survey design of the NHANES data sets (i.e. weight, strata and cluster variables) and computed descriptive statistics including proportions and means for each of the independent, dependent and controlling 
Table 1. Means ${ }^{\mathrm{a}}$ and standard errors of study measures.

\begin{tabular}{|c|c|c|c|c|c|c|c|c|}
\hline & \multicolumn{2}{|c|}{ NHANES III } & \multicolumn{2}{|c|}{ NHANES 1999-2004 } & \multicolumn{2}{|c|}{ NHANES 2009-2012 } & \multirow[b]{2}{*}{ Min } & \multirow[b]{2}{*}{ Max } \\
\hline & Mean & SE & Mean & SE & Mean & SE & & \\
\hline \multicolumn{9}{|l|}{ Body measures $^{b}$} \\
\hline \multicolumn{9}{|l|}{ Bone length } \\
\hline Standing height & 169.79 & 0.20 & 170.38 & 0.20 & 170.30 & 0.21 & 131.60 & 206.50 \\
\hline Upper-leg length & 41.06 & 0.14 & 41.40 & 0.10 & 39.69 & 0.08 & 23.00 & 55.50 \\
\hline Upper-arm length & 36.16 & 0.05 & 37.61 & 0.07 & 37.38 & 0.06 & 25.20 & 47.70 \\
\hline Waist-to-hip ratio & 0.89 & 0.00 & - & - & - & - & 0.51 & 1.95 \\
\hline Biacromial breadth & 39.22 & 0.08 & - & - & - & - & 29.40 & 53.30 \\
\hline Biiliac breadth & 28.92 & 0.08 & - & - & - & - & 17.30 & 48.70 \\
\hline \multicolumn{9}{|l|}{ Girth } \\
\hline Body weight & 75.75 & 0.39 & 81.08 & 0.44 & 82.80 & 0.44 & 34.75 & 241.80 \\
\hline Body-mass index & 26.18 & 0.13 & 27.84 & 0.13 & 28.47 & 0.17 & 14.30 & 84.87 \\
\hline Waist circumference & 89.63 & 0.27 & 94.82 & 0.31 & 96.52 & 0.42 & 58.60 & 174.10 \\
\hline Arm circumference & 31.85 & 0.11 & 33.03 & 0.10 & 33.37 & 0.13 & 18.30 & 61.00 \\
\hline Thigh circumference & 52.29 & 0.13 & 54.63 & 0.15 & - & - & 32.00 & 100.00 \\
\hline Elbow breadth & 6.86 & 0.01 & - & - & - & - & 4.10 & 10.40 \\
\hline Wrist breadth & 5.49 & 0.01 & - & - & - & - & 4.00 & 7.40 \\
\hline Buttocks circumference & 100.64 & 0.24 & - & - & - & - & 68.50 & 179.20 \\
\hline Calf circumference & - & - & 39.20 & 0.10 & - & - & 20.70 & 70.50 \\
\hline T. gondii seropositive & 0.18 & 0.01 & 0.12 & 0.01 & 0.09 & 0.01 & 0.00 & 1.00 \\
\hline \multicolumn{9}{|l|}{ Controls } \\
\hline Age (in years) & 34.32 & 0.21 & 35.69 & 0.20 & 35.28 & 0.26 & 21.00 & 49.00 \\
\hline Female & 0.50 & 0.01 & 0.50 & 0.01 & 0.51 & 0.01 & 0.00 & 1.00 \\
\hline \multicolumn{9}{|l|}{ Race-ethnicity } \\
\hline Non-Hispanic white & 0.75 & 0.01 & 0.73 & 0.02 & 0.68 & 0.03 & 0.00 & 1.00 \\
\hline Non-Hispanic black & 0.11 & 0.01 & 0.12 & 0.01 & 0.12 & 0.01 & 0.00 & 1.00 \\
\hline Mexican American & 0.06 & 0.01 & 0.10 & 0.01 & 0.12 & 0.02 & 0.00 & 1.00 \\
\hline Other & 0.08 & 0.01 & 0.04 & 0.01 & 0.08 & 0.01 & 0.00 & 1.00 \\
\hline Education (in years) & 12.89 & 0.09 & 0.00 & 0.00 & 0.00 & 0.00 & 0.00 & 17.00 \\
\hline \multicolumn{9}{|l|}{ Education } \\
\hline No high school diploma & - & - & 0.15 & 0.01 & 0.14 & 0.01 & 0.00 & 1.00 \\
\hline High school diploma & - & - & 0.26 & 0.01 & 0.20 & 0.01 & 0.00 & 1.00 \\
\hline More than high school & - & - & 0.59 & 0.01 & 0.66 & 0.02 & 0.00 & 1.00 \\
\hline Poverty-to-income ratio & 3.05 & 0.07 & 3.03 & 0.05 & 2.87 & 0.07 & 0.00 & 11.29 \\
\hline CMV seropositive & 0.55 & 0.01 & 0.53 & 0.02 & 0.00 & 0.00 & 0.00 & 1.00 \\
\hline
\end{tabular}

$\mathrm{CMV}$ - cytomegalovirus; SE - Standard error; ${ }^{\text {a }}$ proportions reported for categorical variables $\left(\mathrm{kg} / \mathrm{m}^{2}\right)$; ${ }^{\mathrm{b}}$ in centimetres with the exception of weight $(\mathrm{kg})$ and body-mass index $\left(\mathrm{kg} / \mathrm{m}^{2}\right)$. Sample sizes: NHANES III = 7118, 1999-2004 = 4008, 2009-2012=4036.

variables used in our analyses. We obtained estimates of the relationship between body measures and latent toxoplasmosis using ordinary least-squares (OLS) regression, including sex, race-ethnicity, CMV seropositivity, education, age and PIR as covariates in each model with the only exception being CMV, which was not available in the 2009-2012 NHANES data set. We repeated these same analyses with male-only and female-only subgroups to examine potential sex-specific effects.

We used OLS regression to test for an association between infection with $T$. gondii and testosterone concentration. We further tested for potential interactions between latent toxoplasmosis and testosterone concentration in the prediction of each available body measure. As sufficient testosterone data were only available for subjects in the 2011-2012 NHANES data set, we were limited to this subsample for each analysis. In each of these analyses, we evaluated both main effects and interactions for both the full sample as well as for male and female subsamples.

\section{RESULTS}

Among the three NHANES data sets analysed in the present study, infection with $T$. gondii ranged from $18 \%$ (NHANES III) to 9\% (2009-2012 NHANES), indicating a declining prevalence of latent toxoplasmosis in the US over the past two decades. The number of men and women included in each NHANES sample were nearly equal, and the average age was approximately 34 years. The majority of subjects were non-Hispanic white (range 68-75\%), had an education greater than high school and had an average PIR of approximately 3 (Table 1).

In each of the three NHANES data sets used in this study, there were no significant differences between seropositive and seronegative groups for any of the body measures examined (Table 2). Results did not differ when limiting the models to either women (Table 3 ) or men (Table 4). As the differences in findings between these analyses and those performed by Flegr et al. (2005) could potentially be attributed to use of different covariates, we did the same analyses again with only age, sex and CMV infection status (where data were available) as covariates to more closely match the methodology used by Flegr et al. (2005). In these partially controlled models, we found significant associations between infection with $T$. gondii and reduced height in both men and women across each of the NHANES data sets (Tables S1-S3).

We found no significant associations between infection with $T$. gondii and testosterone concentration in either the 
Table 2. Body measures ${ }^{\text {a }}$ and Toxoplasma gondii seropositivity in 21 to 49 -year-old U.S. adults: unstandardised coefficients and standard errors from ordinary least-squares regression.

\begin{tabular}{lcccccc}
\hline & \multicolumn{2}{c}{ NHANES III } & \multicolumn{2}{c}{ NHANES 1999-2004 } & \multicolumn{2}{c}{ NHANES 2009-2012 } \\
\cline { 2 - 7 } & $\beta$ & SE & $\beta$ & SE & $\beta$ & SE \\
\hline Bone length & & & & & & \\
Standing height & -0.229 & 0.271 & -0.583 & 0.408 & -0.368 & 0.531 \\
Upper-leg length & -0.071 & 0.135 & -0.213 & 0.179 & 0.007 & 0.207 \\
Upper-arm length & -0.005 & 0.093 & -0.069 & 0.135 & 0.120 & 0.179 \\
Waist-to-hip ratio & 0.003 & 0.003 & - & - & - & - \\
Biacromial breadth & -0.012 & 0.123 & - & - & - & - \\
Biiliac breadth & -0.058 & 0.136 & - & - & - & - \\
Girth & & & & & & \\
Body weight & 0.165 & 0.786 & -1.739 & 1.108 & -0.247 & 1.567 \\
Body-mass index & 0.097 & 0.255 & -0.416 & 0.310 & 0.066 & 0.487 \\
Waist circumference & 0.215 & 0.627 & -1.108 & 0.754 & -0.079 & 1.185 \\
Arm circumference & -0.104 & 0.211 & -0.436 & 0.267 & -0.043 & 0.382 \\
Thigh circumference & 0.036 & 0.280 & -0.673 & 0.364 & - & - \\
Elbow breadth & -0.004 & 0.022 & - & - & - & - \\
Wrist breadth & -0.009 & 0.015 & - & - & - & - \\
Buttocks circumference & -0.150 & 0.519 & - & - & - & - \\
Calf circumference & - & - & -0.361 & 0.251 & - & - \\
\hline
\end{tabular}

Each body measure was examined as a separate model. All models include sex, age, race-ethnicity, education and poverty-to-income ratio as controls. None of the coefficients in the table is significantly different from zero. ${ }^{a}$ all dependent variables measured in centimetres with the exception of weight $(\mathrm{kg})$ and body-mass index $\left(\mathrm{kg} / \mathrm{m}^{2}\right)$. Sample sizes: NHANES III $=7118,1999-2004=4008,2009-2012=4036$.

Table 3. Body measures ${ }^{\mathrm{a}}$ and Toxoplasma gondii seropositivity in female 21 to 49 -year-old U.S. adults: unstandardised coefficients and standard errors from ordinary least-squares regression.

\begin{tabular}{lcccccc}
\hline & \multicolumn{2}{c}{ NHANES III } & \multicolumn{2}{c}{ NHANES } & 1999-2004 & \multicolumn{2}{c}{ NHANES 2009-2012 } \\
\cline { 2 - 8 } & $\beta$ & SE & $\beta$ & SE & $\beta$ & SE \\
\hline Bone length & & & & & & \\
Standing height & -0.556 & 0.360 & -0.783 & 0.495 & -0.133 & 0.679 \\
Upper-leg length & -0.026 & 0.228 & -0.357 & 0.254 & -0.047 & 0.243 \\
Upper-arm length & -0.146 & 0.114 & -0.240 & 0.188 & 0.220 & 0.247 \\
Waist-to-hip ratio & 0.000 & 0.004 & - & - & - & - \\
Biacromial breadth & -0.026 & 0.160 & - & - & - & - \\
Biiliac breadth & -0.372 & 0.218 & - & - & - & - \\
Girth & & & & & & \\
Body weight & -0.586 & 1.151 & -1.310 & 1.557 & -0.884 & 1.843 \\
Body-mass index & -0.080 & 0.431 & -0.255 & 0.519 & -0.140 & 0.688 \\
Waist circumference & -0.551 & 0.913 & -0.863 & 1.159 & -0.545 & 1.488 \\
Arm circumference & -0.070 & 0.347 & -0.317 & 0.404 & -0.194 & 0.517 \\
Thigh circumference & 0.059 & 0.463 & -0.805 & 0.523 & - & - \\
Elbow breadth & -0.041 & 0.030 & - & - & - & - \\
Wrist breadth & -0.027 & 0.020 & - & - & - & - \\
Buttocks circumference & -0.596 & 0.885 & - & - & - & - \\
Calf circumference & - & - & -0.256 & 0.349 & - & - \\
\hline
\end{tabular}

Each body measure was examined as a separate model. All models include sex, age, race-ethnicity, education and poverty-to-income ratio as controls.

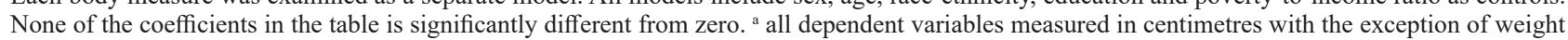
$(\mathrm{kg})$ and body-mass index $\left(\mathrm{kg} / \mathrm{m}^{2}\right)$. Sample sizes: NHANES III = 3828, 1999-2004 =2 139, 2009-2012 = 2105 .

full sample $(\beta=0.02[-0.06,0.09] ; p=0.614)$ or for men $(\beta=-0.04[-0.13,0.06] ; p=0.410)$ or women $(\beta=0.08$ $[-0.05,0.21] ; p=0.228)$ considered separately. In testing for potential interactions between latent toxoplasmosis and testosterone concentration, we found no significant interactions in the prediction of each body measure available in the 2011-2012 data set. Again, to provide a more direct replication of Flegr et al. (2005), we chose to repeat these analyses with only age and sex (CMV was unavailable in the 2011-2012 data set) included as covariates but again found no significant interactions in this reduced model.

\section{DISCUSSION}

Our findings represent a comprehensive investigation of associations between latent toxoplasmosis and multiple body measures in three large data sets representative of the US population. In 2005, Flegr et al. reported that seropositive men were taller than seronegative men but found no association between seropositivity and height in women. In contrast, in our partially controlled models using the same covariates used by Flegr et al. (2005), we found that infection with $T$. gondii was associated with shorter standing height in both women and men. However, in the models in- 
Table 4. Body measures ${ }^{\mathrm{a}}$ and Toxoplasma gondii seropositivity in male 21 to 49 -year-old U.S. adults: unstandardised coefficients and standard errors from ordinary least-squares regression.

\begin{tabular}{lcccccc}
\hline & \multicolumn{2}{c}{ NHANES III } & \multicolumn{2}{c}{ NHANES } & 1999-2004 & \multicolumn{2}{c}{ NHANES 2009-2012 } \\
\hline & $\beta$ & SE & $\beta$ & SE & $\beta$ & SE \\
\hline Bone length & & & & & & \\
Standing height & 0.114 & 0.374 & -0.406 & 0.558 & -0.609 & 0.810 \\
Upper-leg length & -0.109 & 0.163 & -0.097 & 0.206 & 0.043 & 0.310 \\
Upper-arm length & 0.131 & 0.134 & 0.100 & 0.176 & 0.021 & 0.250 \\
Waist-to-hip ratio & 0.006 & 0.005 & - & - & - & - \\
Biacromial breadth & 0.011 & 0.133 & - & - & - & - \\
Biiliac breadth & 0.244 & 0.159 & - & - & - & - \\
Girth & & & & & & \\
Body weight & 0.923 & 1.072 & -1.652 & 1.445 & 0.188 & 2.144 \\
Body-mass index & 0.277 & 0.293 & -0.388 & 0.400 & 0.202 & 0.534 \\
Waist circumference & 0.935 & 0.880 & -0.859 & 0.968 & 0.218 & 1.538 \\
Arm circumference & -0.101 & 0.246 & -0.410 & 0.335 & 0.074 & 0.401 \\
Thigh circumference & 0.059 & 0.329 & -0.450 & 0.478 & - & - \\
Elbow breadth & 0.035 & 0.030 & - & - & - & - \\
Wrist breadth & 0.011 & 0.021 & - & - & - & - \\
Buttocks circumference & 0.327 & 0.643 & - & - & - & - \\
Calf circumference & - & - & -0.377 & 0.281 & - & - \\
\hline
\end{tabular}

Each body measure was examined as a separate model. All models include sex, age, race-ethnicity, education and poverty-to-income ratio as controls. None of the coefficients in the table is significantly different from zero. ${ }^{\text {a }}$ all dependent variables measured in centimetres with the exception of weight $(\mathrm{kg})$ and body-mass index $\left(\mathrm{kg} / \mathrm{m}^{2}\right)$. Sample sizes: NHANES III = 3290, 1999-2004 = 1 869, 2009-2012=1931.

corporating additional covariates, we found no significant associations between latent toxoplasmosis and height for either men or women in any of the NHANES data sets included in this study. Further, we found no significant associations for any of the other body measures assessed. These findings show no evidence that infection with $T$. gondii is associated with adult body morphology in either women or men, even when considering interactions between seropositivity and testosterone concentration.

We also found no evidence that latent toxoplasmosis is associated with testosterone concentration in either women or men, suggesting that in these samples latent toxoplasmosis does not affect testosterone concentration in either women or men and that adult testosterone concentration does not increase the risk of latent toxoplasmosis in either men or women. However, because we did not have data about the ratio of the lengths of the second and fourth digits of the hand, we could not test the hypothesis that prenatal androgenisation is associated with exposure to $T$. gondii (see Flegr et al. 2005).

It is possible that the differences in findings between the report that showed an association between infection with T. gondii and height in men (Flegr et al. 2005) and this study could be due to differing sample characteristics such as the geographic region in which samples were obtained, age of exposure, sample size or strain of T. gondii. Further, dissimilarities in demographic characteristics could, in part, explain some of the differences in findings between this study and the findings reported by Flegr et al. (2005). For example, our sample was, on average, older (mean age $=34.32$ years) than the average of 21 years in the Flegr et al. (2005) study. Still, within our data set, we did not find an age effect.

Beyond sampling differences, the two studies also differ in the covariates included in the statistical mod- els. Both Flegr et al. (2005) and the present study included age, sex and cytomegalovirus seropositivity as controlling covariates. Indeed, including only these covariates in our own analyses, we did detect significant differences in height in both men and women, though both women and men with latent toxoplasmosis were shorter than those without latent toxoplasmosis. In the present study, we also included educational attainment, PIR and race-ethnicity as covariates. Prior studies suggest that socioeconomic factors such as educational attainment and income level may impact morphological development, especially in children (Finch and Beck 2011, Tucker-Seeley and Subramanian 2011, Do et al. 2013). In addition, average body morphology differs across race-ethnicities (Ousley et al. 2009), as does the prevalence of infection with T. gondii (McQuillan et al. 2004). Therefore, we felt the inclusion of these additional covariates to be important for improved generalisation of the results. Finally, strains of T. gondii differ in virulence (Song et al. 2013), potentially limiting comparison of our findings with other studies in that the data sets we used did not enable us to determine strain of $T$. gondii.

This study has several limitations. First, the cross-sectional nature of the data did not enable determination of the time of infection with $T$. gondii. Infection at certain ages may affect body morphology more than infection at other ages. Similarly, though we found no association between seropositivity and testosterone concentration, there was no way to monitor the potential effect of $T$. gondii on testosterone over time. Another limitation is the absence or difference of some body measures between the NHANES III and continuous NHANES sample groups. These differences make direct comparisons between each of the NHANES data sets more difficult. Further, data were not available for $2 \mathrm{D}: 4 \mathrm{D}$ finger length ratio in any of the NHANES data 
sets. It is possible that this measure used by Flegr et al. (2005) may better estimate prenatal androgenisation than did the measures we used. Finally, we were unable to identify particular strains of $T$. gondii.

The present study also possesses a number of important strengths. First, by utilising data from both the NHANES III and continuous NHANES data sets, we were able to utilise a large sample size that spanned multiple decades and increased statistical power to detect differences in morphology between subject seropositive and seronegative for T. gondii. That is, were there associations between T. gondii and height, we would have been more likely to detect them due to greater statistical power. Further, use of the NHANES data sets enabled the inclusion of a number of body measures not previously investigated for potential associations with $T$. gondii infection, such as biacromial breadth, calf circumference, and several others.

In conclusion, in this study using multiple large NHANES data sets and including numerous human body measures, we found no evidence for an association between infection with $T$. gondii and measures of human morphology. Further, there were no significant interactions between seropositivity and testosterone in the prediction of multiple body measures. Together, these findings suggest that in the USA, latent toxoplasmosis is not associated with human morphology, including height. Future research might consider the use of longitudinal methods to better investigate associations between the time of infection with $T$. gondii and human morphology and whether specific strains of T. gondii might be associated with human morphology.

\section{REFERENCES}

Do D.P., Watkins D.C., Hiermeyer M., Finch B.K. 2013: The relationship between height and neighborhood context across racial/ethnic groups: a multi-level analysis of the 1999-2004 U.S National Health and Nutrition Examination Survey. Econ. Hum. Biol. 11: 30-41.

Finch B.K., BECK A.N. 2011: Socio-economic status and z-score standardized height-for-age of U.S.-born children (ages 2-6). Econ. Hum. Biol. 9: 272-276.

Finsterer J., Auer H. 2013: Parasitoses of the human central nervous system. J. Helminthol. 87: 257-270.

Flegr J. 2013: How and why Toxoplasma makes us crazy. Trends Parasitol. 29: 156-163.

Flegr J., Hruková M., Hodný Z., Novotná M., Hanušová J. 2005: Body height, body mass index, waist-hip ratio, fluctuating asymmetry and second to fourth digit ratio in subjects with latent toxoplasmosis. Parasitology 130: 621-628.

Flegr J., Kodym P., Tolarová V. 2000: Correlation of duration of latent Toxoplasma gondii infection with personality changes in women. Biol. Psychol. 53: 57-68.

Flegr J., Lindová J., Kodym P. 2008: Sex-dependent toxoplasmosis-associated differences in testosterone concentration in humans. Parasitology 135: 427-431.

Gale S.D., Brown B.L., Erickson L.D., Berrett A., Hedges D.W. 2015: Association between latent toxoplasmosis and cognition in adults: a cross-sectional study. Parasitology: 142: 1-9.

Gunter E.W., Lewis B.G., Koncikowski S.M. 1996: Laboratory Procedures Used for the Third National Health and Nutrition Examination Survey (NHANES III), 1988-1994, Centers for Disease Control and Prevention, U.S. Department of Health and Human Services, Hyattsville, Maryland, pp. 722-729.
Hodková H., Kolbeková P., Skallová A., Lindová J., Flegr J. 2007: Higher perceived dominance in Toxoplasma infected men - a new evidence for role of increased level of testosterone in toxoplasmosis-associated changes in human behavior. Neuro. Endocrinol. Lett. 28: 110-114.

Jones J.L., Kruszon-Moran D., Rivera H.N., Price C., Wilkins P.P. 2014: Toxoplasma gondii seroprevalence in the United States 2009-2010 and comparison with the past two decades. Am. J. Trop. Med. Hyg. 90: 1135-1139.

Kaňková S., Flegr J., CALDA P. 2015: An elevated blood glucose level and increased incidence of gestational diabetes mellitus in pregnant women with latent toxoplasmosis. Folia Parasitol. 62: 056.

McQuillan G.M., Kruszon-Moran D., Kottiri B.J., CurTin L.R., Lucas J.W., Kington R.S. 2004: Racial and ethnic differences in the seroprevalence of 6 infectious diseases in the United States: data from NHANES III, 1988-1994. Am. J. Publ. Hlth. 94: 1952-1958.

Mortensen P.B., Norgaard-Pedersen B., Waltoft B.L., SoRensen T.L., HougaArd D., Yolken R.H. 2007: Early infections of Toxoplasma gondii and the later development of schizophrenia. Schizophr. Bull. 33: 741-744.

Ousley S., JAntz R., Freid D. 2009: Understanding race and human variation: why forensic anthropologists are good at identifying race. Am. J. Phys. Anthropol. 139: 68-76.

Song C., Chiasson M.A., Nursimulu N., Hung S.S., Wasmuth J., Grigg M.E., PARkinson J. 2013: Metabolic reconstruction identifies strain-specific regulation of virulence in Toxoplasma gondii. Mol. Syst. Biol. 9: 708.

Tucker-Seeley R.D., Subramanian S.V. 2011: Childhood circumstances and height among older adults in the United States. Econ. Hum. Biol. 9: 194-202. 